\title{
HISTOLOGICAL EVALUATION OF ENHANCED OSTEO-INDUCTION BY NANO-STRUCTURED TITANIUM GRANULES
}

\author{
Mohamed AbdElaziz Ghanem*, Maha Hakam**, \\ Sameh Mekhemer** and Mohamed Abdel-Mottaleb***
}

\begin{abstract}
Technological advancement in addition to better understanding of bone-healing biology, however, have recently lead to the development of several bone graft substitutes that are currently available to surgeons. In the current study we introduce a new bone grafting material, its structure is based on pure commercially available nano-particles of Titanium Dioxide $\left(\mathrm{TiO}_{2}\right)$. This study aims to evaluate this $\mathrm{TiO}_{2}$ bone graft substitute interaction with viable bone histologically by assessing its osteo-induction and conduction properties.
\end{abstract}

KEYWORDS: TiO2 - nanotechnology - bone grafting - osteoinduction - osteoconduction calvaria - albino rat - H\&E

\section{INTRODUCTION}

Bone tissue possesses a high capacity of spontaneous regeneration, Nevertheless, this potential is limited by the size of the defect. Thus, very extensive defects such as those caused by trauma due to a car accident or after removal of a large pathology represent a problem to the orthopedic and/or the oromaxillofacial surgeon. Over the last few years, various surgical procedures using biological or synthetic bone substitutes have been developed for the treatment of these defects in an attempt to promote their closure with new bone tissue that possesses morphological and functional characteristics similar to those of the original tissue.

Bone graft is the second most common transplantation tissue, with blood being by far the commonest. ${ }^{[1]}$ More than 500,000 bone grafting procedures are happening annually in the United States and 2.2 million worldwide in order to repair bone defects in orthopedics, neurosurgery and dentistry.

The two main reasons to use bone grafts are to stimulate the bone to heal and to provide support to the skeleton by filling gaps between two bones. A bone graft substitute is used like 'fertilizer' to stimulate and speed the bone healing process.

B.D.S. 2006 (October 6 University).

Professor of Oral and Maxillofacial Surgery, Faculty of Oral and Dental Medicine, Cairo University.

Associate Professor School of Engineering \& Applied Sciences Director, Centre for Nano Technology, Nile University. 
Considerable information is available in the literature on bone morphogenesis, growth, remodeling and repair. ${ }^{[1]}$ Successful bone healing and repair requires a coordinated and complex transcriptional program that integrates mechanical stimulus, signaling, angiogenesis and osteogenesis. However, the interdependence of these processes is not fully understood. Bone healing entails an environment characterized by a supportive scaffold within and upon which pluri-potent stem cells undergo proliferation and differentiation. ${ }^{[1]}$

Repair activities depend upon the temporal and spatial distribution of appropriate osteogenic molecules, including extracellular matrix proteins. Given the enormous biomedical burden of skeletal injury, and the need to treat congenital, posttraumatic and post-surgical conditions in which bone regeneration is unsuccessful, understanding osteogenesis for skeletal repair has important clinical implications..$^{[2,3]}$

When bone is fractured or there is a gap produced as a result of tissue loss due to surgical removal, cells and the cell mediators, in the form of extracellular signals (mechanical and chemical), collectively ensure the restoration of form and function. Bone healing is a complex physiological process which involves the coordination of several different cell types. Cell activities bear partial resemblance to the molecular cascade characterizing natal bone formation. ${ }^{[3]}$

The ideal bone substitute should possess the following characteristics. ${ }^{[4,5]}$

1. It should be biologically compatible.

2. Non-supportive for local pathogens or cross infection.

3. Osteogenic (facilitate bone cell ingrowths).

4. Osteoinductive (stimulates non-differentiated mesenchymal cells to form bone cells).

5. Osteoconductive (serves as a scaffold for the ingrowth of capillaries, perivascular tissue and osteoprogenitor cells from the recipient bed).

6. Should match the physical and chemical composition of natural bone trabeculae.

7. Should be resorbable and Osteotropic (enhance bone formation by its chemical or structural characteristics.

8. Should provide Calcium and Phosphate sources.

9. Should be micro-porous and easy to handle.

10. Exist in unlimited supply without need for violation of distant donor.

11. Should be completely host bone of the same or superior quality and quantity as quickly as possible.

12. Maintains the mechanical stability and volume during the initial healing phase and then subsequently resorbs completely and being replaced by newly formed bone.

\section{The aim of the study:}

Histological assessment of the osteo-inductive and conductive properties of nano-structured titanium granules as a bone grafting material.

\section{MATERIALS}

\section{$\mathrm{TiO}_{2}$ granules Manufacturing:}

Commercially available pure Titanium Dioxide $\left(\mathrm{TiO}_{2}\right)$ nano-particles (P25 DeGussa - Hüls AG Frankfurt, Germany), supplied from Nanotechnology Centre, Nile University, Cairo, Egypt, was used to produce the granules.

In order to produce a super saturated solution, more material is added till a slurry of a creamy consistency is produced, the resultant slurry is put in a hot furnace (Tanita wide oven 220V-620W, Japan) for vaporization of the liquid part, the resultant material is like a chalky white small porous rocks, that were crushed manually into smaller sizes, 
passed through series of sieves to separate the required size of granules (size $\sim 0.30-0.70 \mathrm{~mm}$ ).

\section{Animals}

Twelve male albinoWistar rats aged around 100 days with body mass around $300 \mathrm{~g}$ in controlled breeding conditions, kept in a certified animal house, were selected for the experiment. Temperature: $(22 \pm 2)^{\circ} \mathrm{C}$, relative humidity: $(55 \pm 10) \%$, light/dark hours schedule : 12/12. Individual body weights were recorded prior to implantation and thereafter on scarification.

\section{Grouping}

The animals were divided into two groups.

- Group I (Control Group 6 animals) the defects were left to be only filled with blood clots.

- Group II (study group, 6 animals) the defect was filled with $\mathrm{TiO}_{2}$ graft soaked in the animal's own blood.

\section{Animal anesthesia}

All animals were submitted to skull surgery under general anesthesia with intramuscular injection of Xylazine / Ketamine \{Xylazine $6 \mathrm{mg} /$ $\mathrm{kg}$ and Ketamine $70 \mathrm{mg} / \mathrm{kg}\}^{[6,7]}$

\section{Surgical procedure}

After shaving the fronto-parietal region and vigorous disinfection with Povidone-iodine and Isolation of the surgical area, a semi-lunar shaped incision was made in the scalp in the anterior region of the calvarium allowing reflection of a full-thickness flap in a backwards direction, a \#15 surgical knife was used.

\section{Animal scarification}

Two rats from each group was sacrificed with an overdose of Kitamine double LD50 Dose (IP $229 \mathrm{mg} / \mathrm{kg}$ ) over a period of 6 months at intervals of 2, 4 and 6 months, and submitted to histological examinations.

\section{RESULTS}

Throughout the experiment, five animals died, three of them, death occurred intra-operatively due to sudden penetration of the trephine bur into brain tissues, due to tearing of the dura matter, or due to excessive bleeding intra-operative. Two of the animals died within the 1st two weeks post-operatively. However, any animal loss was compensated with a new animal to keep the number of experimental rats to twelve.

\section{A. Gross Findings}

Postoperative healing was uneventful in general for both study group and control one. Healing was free of complications and no signs of post operative infection or exposure of bone materials in the clinical observation were reported in the study group.

\section{B. Histological evaluation}

At 2 months, the defect sites were mostly filled with fibrous connective tissue rich with fibroblasts. No new bone formation was observed. The defect was filled with connective tissue.

At 4 months, in the control group only very few sections showed that new bone was formed very thinly around the margins of the defect on the normal bone, and no new bone formation was observed in the central part of the defect area.

\section{Control group (non grafted group) at 6 months}

\section{2. $\mathrm{TiO}_{2}$ Study group}

\section{1) 2 months healing period \\ 2) 4 months healing period \\ 3) 6 months healing period}

\section{Toxicological effects of $\mathrm{TiO}_{2}$ graft:}

Toxicological effects of the $\mathrm{TiO}_{2}$ granules on the selected animal model, by means of penetration of nearby tissue -here were the brain meningies and scalp layers- and accumulation in main body organs 
liver, spleen, heart and kidneys. all showed no toxic effect at all.

\section{DISCUSSION}

The present animal study was designed to evaluate the histological pattern of healing in experimentally induced cranial defects in Albino rats, treated with $\mathrm{TiO}_{2}$ granules bone graft material in comparison to a control model where no graft material was used at all.

For this work the calvarial rat model was chosen because it is well-documented as a model for evaluating bone healing, easy to manipulate, there is no need to stabilize the wound, no muscular layers, the animal is easy to house and feed and less expensive ${ }^{[8]}$ A critical size defect in animal models has been defined as the smallest intraosseous wound that does not heal by bone formation during the lifetime of the animal. Most notably, critical size defects have been of critical importance to the testing of biomaterials. ${ }^{[8,9]}$

On the use of calvarial defects, it was chiefly because most of the calvarial and facial bones have a similar embryologic origin and their bone formation occurs by membranous ossification. Defects of $8 \mathrm{~mm}$ created surgically in rat's calvaria by Takagi and Urist in $1982^{[10]}$ showed healing by connective fibrous tissue after 6 months. Hollinger and Kleinschmidt in $1990^{[9]}$ found that after 13 months of healing, an $8 \mathrm{~mm}$ cranial defect in LongEvans rats did not heal spontaneously. Based on these results, the present study used full-thickness, $8 \mathrm{~mm}$ calvarial defects as they have been shown to fulfill the criteria of a critical size bone defect in albino rats. ${ }^{[11]}$

Although, according to Bosch et al. 1998 ${ }^{[11]}$ who indicated that a unilateral full-thickness 5-mm calvarial defects fulfill the criteria for a critical-size bone defect, which allows for an experiment with a paired design, it avoids inclusion of the sagittal suture in the osseous defect, and it thereby minimizes morbidity by reducing the risk of damaging the mid-sagittal sinus, and avoiding introducing the connective tissue of the suture in which may affect the evaluation of bone regeneration. However, Because of the size of the calvaria of the animals used in the present study, the experimental defect could not be made only in parietal bone. Therefore, a location involving the sagittal suture was chosen in order to standardize the experimental model. Any possible effect from the inclusion of connective tissue of the sagittal suture would be present in all groups.

\section{CONCLUSIONS}

Within the limits of this study, it can be concluded that:

$\mathrm{TiO}_{2}$ granules used as a bone grafting material maintained the volume and contour of the grafted area.

$\mathrm{TiO}_{2}$ granules used as bone grafting material is biocompatible, did not provoke any immunological or toxic reactions.

$\mathrm{TiO}_{2}$ granules hasosteocoductive properties and may have osteoinductivepotential.

Further studies and experiments will be needed to enhance the physical properties of the $\mathrm{TiO}_{2}$ granules to be ready for use as a cheap, easy to use artificial bone graft substitute.

\section{REFERENCES}

1. Doll, B., M. Aleef, and J.O. Hollinger, Overview of fracture repair. Musculoskeletal Tissue Regeneration, 2008: p. 39-61.

2. Jay, R., D. Aaron, and A. Thomas, The role of growth factors in the repair of bone biology and clinical applications. The Journal of Bone and Joint Surgery (American), 2002. 84(6): p. 1032-1044.

3. Lieberman, J.R., A. Daluiski, and T.A. Einhorn, The role of growth factors in the repair of bone. Biology and clinical applications. J Bone Joint Surg Am, 2002. 84-A(6): p. 1032-44. 
4. Mauro, F.A., Assessment of biodegradable calcium polyphosphate for bone substitute applications in the healing of the rat calvarium. 1999 .

5. Cypher, T.J. and J.P. Grossman, Biological principles of bone graft healing. J Foot Ankle Surg, 1996. 35(5): p. 413-7.

6. Karwacki, Z., P. Kowiański, and J. Moryś, General anaesthesia in rats undergoing experiments on central nervous system. Folia morphologica, 2001. 60(4): p. 235-234.

7. Karwacki, Z., P. Kowianski, and J. Morys, General anaesthesia in rats undergoing experiments on the central nervous system. Folia Morphol (Warsz), 2001. 60(4): p. 235-42.
8. Schmitz, J.P. and J.O. Hollinger, The critical size defect as an experimental model for craniomandibulofacial nonunions. Clin Orthop Relat Res, 1986(205): p. 299-308.

9. Hollinger, J.O. and J.C. Kleinschmidt, The critical size defect as an experimental model to test bone repair materials. J Craniofac Surg, 1990. 1(1): p. 60-8.

10. Takagi, K. and M.R. Urist, The role of bone marrow in bone morphogenetic protein-induced repair of femoral massive diaphyseal defects. Clin Orthop Relat Res, 1982(171): p. 224-31.

11. Bosch, C., B. Melsen, and K. Vargervik, Importance of the critical-size bone defect in testing bone-regenerating materials. J Craniofac Surg, 1998. 9(4): p. 310-6. 